\title{
Results on MeV-scale dark matter from a gram-scale cryogenic calorimeter operated above ground
}

\section{CRESST Collaboration}

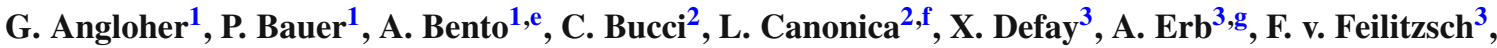 \\ N. Ferreiro Iachellini' ${ }^{1}$, P. Gorla ${ }^{2}$, A. Gütlein ${ }^{4,5}$, D. Hauff ${ }^{1}$, J. Jochum ${ }^{6}$, M. Kiefer ${ }^{1}$, H. Kluck ${ }^{4,5}$, H. Kraus ${ }^{7}$, \\ J.-C. Lanfranchi ${ }^{3}$, A. Langenkämper ${ }^{3}$, J. Loebell ${ }^{6}$, M. Mancuso ${ }^{1}$, E. Mondragon ${ }^{3}$, A. Münster ${ }^{3}$, L. Oberauer ${ }^{3, a}$, \\ C. Pagliarone ${ }^{2}$, F. Petricca ${ }^{1}$, W. Potzel ${ }^{3}$, F. Pröbst ${ }^{1}$, R. Puig ${ }^{4,5}$, F. Reindl ${ }^{1, b}$, J. Rothe ${ }^{1}$, K. Schäffner ${ }^{2, h}$, J. Schieck ${ }^{4,5}$, \\ S. Schönert ${ }^{3}$, W. Seidel ${ }^{1, \mathrm{c}}$, M. Stahlberg ${ }^{4,5}$, L. Stodolsky ${ }^{1}$, C. Strandhagen ${ }^{6}$, R. Strauss ${ }^{1, d}$, A. Tanzke ${ }^{1}$, \\ H. H. Trinh Thi ${ }^{3}$, C. Türkoğlu ${ }^{4,5}$, M. Uffinger ${ }^{6}$, A. Ulrich ${ }^{3}$, I. Usherov ${ }^{6}$, S. Wawoczny ${ }^{3}$, M. Willers ${ }^{3}$, M. Wüstrich ${ }^{1}$, \\ A. Zöller ${ }^{3}$ \\ ${ }^{1}$ Max-Planck-Institut für Physik, 80805 München, Germany \\ ${ }^{2}$ INFN, Laboratori Nazionali del Gran Sasso, 67010 Assergi, Italy \\ ${ }^{3}$ Physik-Department and Excellence Cluster Universe, Technische Universität München, 85747 Garching, Germany \\ ${ }^{4}$ Institut für Hochenergiephysik der Österreichischen Akademie der Wissenschaften, 1050 Vienna, Austria \\ ${ }^{5}$ Atominstitut, Vienna University of Technology, 1020 Vienna, Austria \\ ${ }^{6}$ Eberhard-Karls-Universität Tübingen, 72076 Tübingen, Germany \\ ${ }^{7}$ Department of Physics, University of Oxford, Oxford OX1 3RH, UK
}

Received: 26 July 2017 / Accepted: 11 September 2017 / Published online: 22 September 2017

(c) The Author(s) 2017. This article is an open access publication

\begin{abstract}
Models for light dark matter particles with masses below $1 \mathrm{GeV} / \mathrm{c}^{2}$ are a natural and well-motivated alternative to so-far unobserved weakly interacting massive particles. Gram-scale cryogenic calorimeters provide the required detector performance to detect these particles and extend the direct dark matter search program of CRESST. A prototype $0.5 \mathrm{~g}$ sapphire detector developed for the $v$-cleus experiment has achieved an energy threshold of $E_{t h}=(19.7 \pm 0.9) \mathrm{eV}$. This is one order of magnitude lower than for previous devices and independent of the type of particle interaction. The result presented here is obtained in a setup above ground without significant shielding against ambient and cosmogenic radiation. Although operated in a highbackground environment, the detector probes a new range of light-mass dark matter particles previously not accessi-
\end{abstract}

\footnotetext{
${ }^{a}$ Associated with the CRESST collaboration for this work.

${ }^{b}$ Current address: INFN, Sezione di Roma 1, 00185 Rome, Italy

${ }^{\mathrm{c}}$ Deceased

d e-mail: strauss@mpp.mpg.de

e Also at: Departamento de Fisica, Universidade de Coimbra, 3004516 Coimbra, Portugal

${ }^{f}$ Also at: Massachusetts Institute of Technology, Cambridge, MA 02139, USA

g Also at: Walther-Meißner-Institut für Tieftemperaturforschung, 85748 Garching, Germany

h Also at: GSSI-Gran Sasso Science Institute, 67100 L'Aquila, Italy
}

ble by direct searches. We report the first limit on the spinindependent dark matter particle-nucleon cross section for masses between 140 and $500 \mathrm{MeV} / \mathrm{c}^{2}$.

\section{Introduction}

The origin of dark matter (DM) is still unknown, despite plenty of compelling observational evidence [1]. The most popular model for DM in recent years suggests the existence of weakly interacting massive particles (WIMPs) which are produced in the early Universe [2]. Such particles are in particular appealing since the correct relic density of DM can be produced with a thermally-averaged annihilation cross section $\langle\sigma v\rangle$ which is amazingly close to the weak scale. This natural explanation is referred to as the "WIMP miracle" and has been the main driver for DM physics. Minimal supersymmetric (SUSY) models provide an attractive candidate for WIMPs: the neutralino often assumed as the lightest supersymmetric particle with a mass of $\mathcal{O}\left(1 \mathrm{GeV} / \mathrm{c}^{2}\right)$ to $\mathcal{O}\left(1 \mathrm{TeV} / \mathrm{c}^{2}\right)$ [3]. Lee and Weinberg constrain the mass of WIMPs to $m_{\chi} \geq 2 \mathrm{GeV}$ [4]. Lighter particles would have a too small annihilation cross section $\langle\sigma v\rangle \propto \frac{m_{\chi}^{2}}{m_{Z}^{4}}$ ( $m_{Z}$ : mass of the Z-boson) which would lead to a too large relic abundance 
of DM and, thus, an overclosure of the Universe. Therefore, most experimental DM searches in the past decades focused on GeV-scale particles.

Despite enormous experimental effort, no clear and unambiguous signature for DM has been found by direct, indirect or accelerator searches so far.

Due to this lack of evidence, models which provide alternatives to WIMPs should be probed experimentally. In the past years, rising interest is drawn to light DM (lDM) which extends the allowed mass range of DM particles to $\mathcal{O}(\mathrm{keV})$. Asymmetric DM [5-7], scalar DM particles [8,9] and hidden sector DM [10] are examples for promising and naturally motivated $l \mathrm{DM}$ models. These theories are compatible with the observed relic density (Lee-Weinberg argumentation), the constraints from existing DM searches, in particular indirect searches which are sensitive to $l \mathrm{DM}$, and limits from particle physics experiments.

While searches for axions probe particle masses of keVscale and below, the mass range between $1 \mathrm{MeV}$ and 1000 $\mathrm{MeV}$ is only weakly constrained by $l \mathrm{DM}$-electron scattering [11] or via Bremsstrahlung emission in nuclear recoils [12, 13].

We report here results from the $v$-cleus $0.5 \mathrm{~g}$ prototype detector [14] which enables for the first time to directly probe nuclear recoils induced by DM particles with masses below $500 \mathrm{MeV} / \mathrm{c}^{2}$.

\section{A gram-scale cryogenic calorimeter}

\subsection{Detector technology}

Cryogenic detectors are sensitive to the temperature rise induced by a particle interaction. CRESST-type detectors use single crystals (e.g. $\mathrm{CaWO}_{4}$ or $\mathrm{Al}_{2} \mathrm{O}_{3}$ ) equipped with transition-edge-sensors (TES) made of thin tungsten films. The TES is usually directly coupled to the heat sink by a gold bond wire. Due to the operation at very low temperatures of $\sim 10 \mathrm{mK}$ and the resulting electron-phonon decoupling in the thermometer film, such a cryogenic calorimeter is mainly sensitive to non-thermal phonons [15]. Very low energy thresholds, a high dynamic range (of up to $10^{5}$ ) and a particle-type independent detector response are the main advantages of these devices. State-of-the-art $\mathrm{CaWO}_{4}$ detectors of $300 \mathrm{~g}$ operated in CRESST-II reach thresholds of $\sim 300 \mathrm{eV}$ [16].

The energy threshold of cryogenic detectors depends strongly on the mass $M$ of the crystal which can be quantified by a scaling law discussed in [14]. This describes nicely the performance of existing $\mathrm{CaWO}_{4}$ and $\mathrm{Al}_{2} \mathrm{O}_{3}$ detectors and can be used to predict the sensitivity for detectors of various sizes, materials and geometries [17]. In case of cubic crystals the energy threshold scales as $E_{t h} \propto M^{2 / 3}$ suggesting that thresholds of $10 \mathrm{eV}$ and below are in reach for detectors with masses of $\mathcal{O}(1 \mathrm{~g})$ [14].

\subsection{The prototype calorimeter}

The first prototype calorimeter made of $\mathrm{Al}_{2} \mathrm{O}_{3}$ has a size of $5 \times 5 \times 5 \mathrm{~mm}^{3}$ and a mass of $0.49 \mathrm{~g}$. One side of the optically polished crystal is equipped with a specifically developed TES.

The design is similar to the one used for CRESST light detectors, but adjusted to the size of the crystal [17]. The sensor consists of a $200 \mathrm{~nm}$ thick tungsten film with an area of $0.0061 \mathrm{~mm}^{2}$ and an aluminium phonon collector of thickness $1 \mu \mathrm{m}$ and an area of $0.15 \mathrm{~mm}^{2}$. It is weakly coupled to the heat bath via a gold stripe and a bond wire (heat conductance: $\sim 10 \mathrm{pW} / \mathrm{K}$ at $10 \mathrm{mK})$. The dimension of the TES and the strength of the heat link are chosen such that the life time of non-thermal phonons in the detector $\tau_{n}$ is much shorter than the decay time of the pulses. The former depends on size, geometry and material of the crystal and the TES, the latter is determined by the strength of the heat link to the thermal bath. A detector designed in this way operates in the calorimetric mode [15], i.e. the phonons impinging on the film are integrated over the duration of the non-thermal phonon signal. ${ }^{1}$ A separate gold film sputtered on the crystal acts as an ohmic heater. A current applied to it heats the detector to the desired position in the superconducting phase transition. Artificial pulses of high energy (called control pulses [18]) which saturate the detector response are injected into the heater (here: every $10 \mathrm{~s}$ ). Those allow to control and stabilize the operating point. In addition, artificial pulses of discrete, lower energies (called test pulses) are injected periodically to continuously monitor the detector response (here: every $60 \mathrm{~s})$.

A fit of the pulse model [15] to the pulses of the prototype gram-scale detector confirms its calorimetric operation. The non-thermal phonon life-time in the crystal is $\tau_{n}=(0.30 \pm$ $0.01) \mathrm{ms}$ and the dominant decay time is found to be $\tau_{d e c}=$ (3.64 \pm 0.01$) \mathrm{ms}$ (for the non-thermal pulse component).

Figure 1 (left) shows how the prototype detector is arranged in the experimental setup. The cubic crystal is installed on a copper plate and placed on three sapphire spheres $(\varnothing 1 \mathrm{~mm})$, pressed from the top with another sphere attached to a flexible bronze clamp. The sensor is placed on the top surface where it is electrically and thermally contacted to copper bond pads on the clamp via $\mathrm{Al}$ and $\mathrm{Au}$ wire bonds, respectively. At a distance of about $2 \mathrm{~cm}$ from the crystal, a ${ }^{55} \mathrm{Fe}$ calibration source (activity $\sim 0.2 \mathrm{~Bq}$ ) is installed.

\footnotetext{
${ }^{1}$ Detectors operating in the bolometric mode, in contrast, measure the flux of phonons through the TES [15].
} 

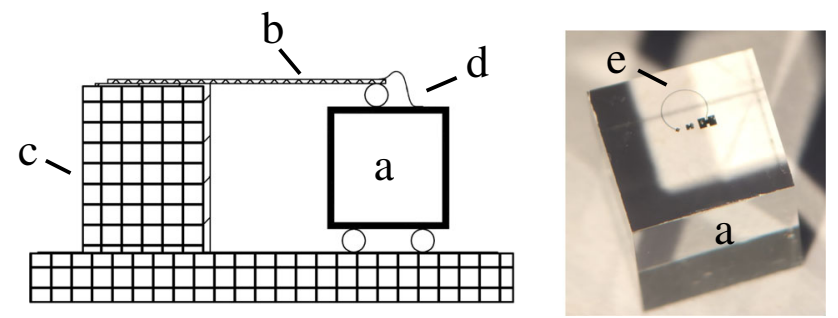

Fig. 1 Left: Schematic drawing of the detector crystal (a) and the copper holder (c). The crystal is placed on $\mathrm{Al}_{2} \mathrm{O}_{3}$ balls and is pressed from top by a flexible bronze clamp (b). Bond wires (d) provide electrical and thermal connection. Right: Picture of the prototype $\mathrm{Al}_{2} \mathrm{O}_{3}$ crystal (a) with the specially designed TES (e). For details see text

\subsection{Experimental setup}

The prototype is operated in a cryostat of the Max-PlanckInstitute for Physics in Munich in a surface building. A commercial dilution refrigerator from Oxford Instruments (Kelvinox 400) is used with a standard helium dewar. The experimental volume is surrounded by a $1 \mathrm{~mm}$ copper thermal screen, but no special care on radiopurity is taken in this setup. No dedicated shielding against ambient radiation is installed within or outside the cryostat. The ceiling of the room is made of $\sim 30 \mathrm{~cm}$ concrete, therefore a moderate reduction of the hadronic component of cosmogenic radiation is expected. In the present setup, no muon veto or other anti-coincidence detectors are installed.

The TES is biased with a constant current $(1.0 \mu \mathrm{A})$ and read out with DC-SQUIDs (Jessy from Supracon). The output of the SQUID electronics is fed to a lownoise voltage amplifier (SR560 from Stanford Research Electronics). For data-taking, two systems have been used in parallel: (1) the standard CRESST data acquisition (DAQ), see e.g. [18], with a hardware trigger unit and 16 bit transient digitizer, and (2) a continuous data-taking system based on a 16 bit digitizer from National Instruments (NI USB-6218 BNC). The latter was used to record a continuous stream of the entire measurement with a sampling frequency of $20 \mathrm{kHz}$. Offline, a software trigger is applied to the data.

\section{Measurement}

The first measurement of the prototype gram-scale calorimeter in the MPI cryostat was primarily intended to de- monstrate an energy threshold in the $10 \mathrm{eV}$ regime for the $v$-cleus experiment [17]. Since a threshold of $\sim 20 \mathrm{eV}$ was reached (see [14] and below), one order of magnitude lower than previous CRESST results [16], this first data can be used to search for new physics: a previously un-explored region of parameter space for DM-particle nucleus scattering can be explored (see Sect. 4). In this section we review the energy

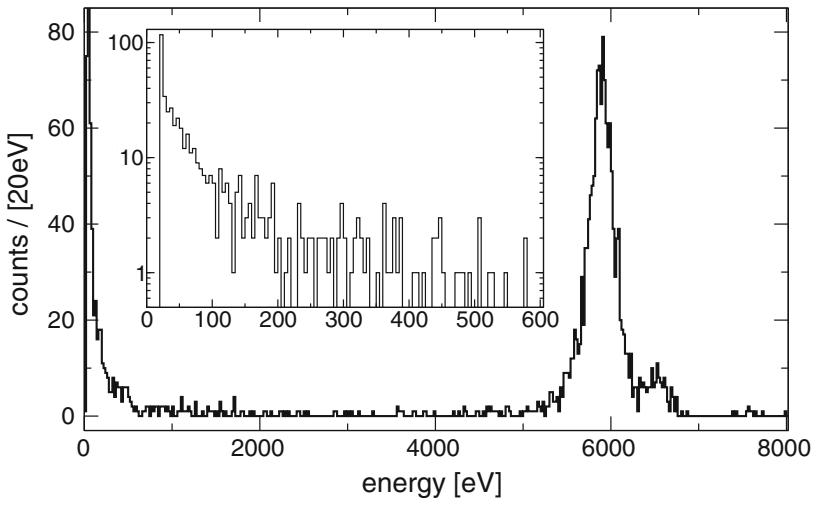

Fig. 2 Total energy spectrum of the $5.3 \mathrm{~h}$ measurement in presence of the ${ }^{55} \mathrm{Fe} \mathrm{X}$-ray source with peaks at 5.90 and $6.49 \mathrm{keV}$. The inset shows the events in the region-of-interest for DM search from the energy threshold of $19.7-600 \mathrm{eV}$ (binning $5 \mathrm{eV}$ ). No data quality cuts are applied

calibration and threshold analysis which is presented in detail in [14], and focus on dedicated aspects of the DM analysis.

\subsection{Energy calibration}

The measurement with the $0.49 \mathrm{~g} \mathrm{Al}_{2} \mathrm{O}_{3}$ prototype calorimeter had a total run-time of $5.31 \mathrm{~h}$ corresponding to an exposure of $0.11 \mathrm{~g}$-days. The ${ }^{55} \mathrm{Fe}$ calibration source was placed in the cryogenic setup during the entire run. The dominant $\mathrm{K}_{\alpha}$ line $\left(E_{\text {lit }}=5.895 \mathrm{keV}\right)$ of ${ }^{55} \mathrm{Mn}$ is used to calibrate the energy spectrum. The linearisation of the pulse response is established by a truncated template fit which uses the pulse shape infomation only in the linear region of the detector response [14]. The method is well established and successfully used in previous CRESST ana- lyses $[16,19]$. The fit reproduces the $\mathrm{K}_{\beta}$ line of ${ }^{55} \mathrm{Mn}$ at an energy of $E_{\text {obs }}=(6.485 \pm 0.017) \mathrm{keV}$, well in agreement with the literature value of $6.490 \mathrm{keV} \mathrm{[14].} \mathrm{The} \mathrm{robustness} \mathrm{of} \mathrm{the} \mathrm{lin-}$ earisation at lower energies is studied and a moderate systematic error of $1.1 \%$ on the energy calibration is derived [14].

Figure 2 (main frame) shows the final energy spectrum with the dominant X-ray lines of ${ }^{55} \mathrm{Mn}$. Above $7 \mathrm{keV}$, a constant background rate of $\sim 1.2 \times 10^{5}$ counts $/(\mathrm{kg} \mathrm{keV}$ day) is observed, while from $\sim 1 \mathrm{keV}$ towards lower energies the spectrum is significantly rising to a rate of about $10^{8}$ counts/[kg keV day] (see discussion below).

\subsection{Software trigger}

For the evaluation of the pulse amplitudes in the linear region of the detector response (up to $600 \mathrm{eV}$ ), the optimum filter is used [20,21]. This method improves the reconstruction of a known signal in the presence of noise with a measured power spectrum and typically gives significantly better results than 


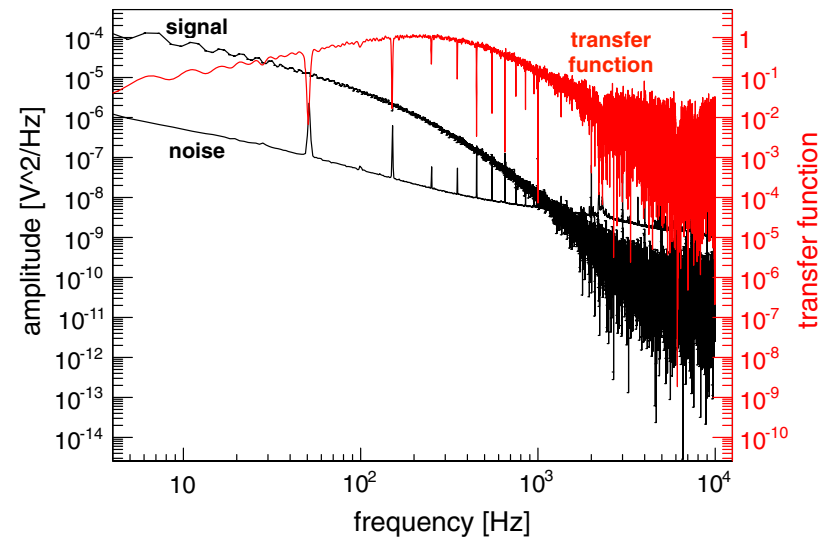

Fig. 3 Left axis: measured power spectra of randomly selected noise traces and of the template pulse (signal). Right axis: the corresponding filter transfer function. See text for details

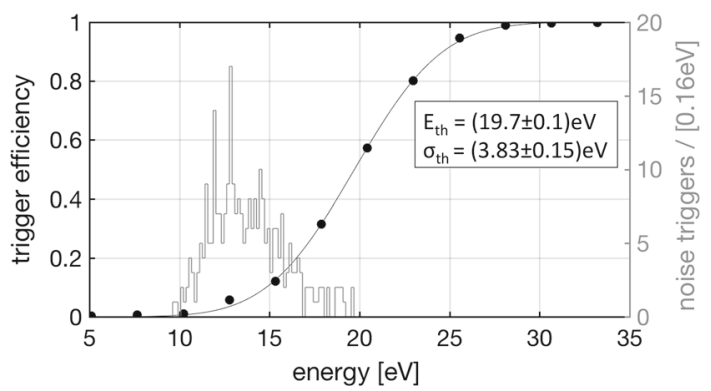

Fig. 4 Trigger efficiency evaluated at discrete energies (dots, left axis) by using the optimum trigger algorithm. These data points are fitted by an error function (solid line, see text). The histogram shows the distribution of the filter output (maximum) of randomly sampled noise traces (right axis). The energy threshold $E_{t h}$ is chosen such that a negligible amount of noise triggers is accepted. Data from [14]

the template fit. The filter transfer function is calculated by the ratio of the power spectra of the template pulse and the noise. This function is used to weight the spectral components of the data sample (in the frequency domain) according to the respective signal-to-noise-ratio. Figure 3 shows the measured power spectra (left axis) and the resulting transfer function (right axis) of the optimum filter used for the analysis of the prototype calorimeter. After being applied in the frequency space, the filter output is transformed back to time domain and normalized to match the maximum of the original pulse. In case of this measurement, a baseline noise of $\sigma_{b}=(3.74 \pm$ $0.21) \mathrm{eV} \mathrm{[14]} \mathrm{is} \mathrm{reached} \mathrm{with} \mathrm{the} \mathrm{optimum} \mathrm{filter,} \mathrm{a} \mathrm{factor} \mathrm{of}$ 1.7 smaller compared to that obtained with the template fit.

The trigger threshold is derived by a systematic study [14]. The optimum filter is applied to a set of noise traces, which are randomly recorded by the (standard) DAQ, and is evaluated at every position within a single trace. The maximum filter output for each trace is stored. Figure 4 shows a histogram of the maximum filter output for $\sim 400$ baseline traces. The threshold of the software trigger has to be cho- sen such that noise triggers are negligible. In the case of this measurement, the trigger threshold is set to $13.0 \mathrm{mV}$ [14]. To derive the trigger efficiency, artificial template pulses of discrete energies are added to the randomly chosen noise traces. After applying the optimum filter, the fraction of events above and below the trigger threshold are calculated for each simulated energy. The result is shown as dots in Fig. 4. These data points are well fit by an error function $p_{t r i g}(E)=$ $0.5 \cdot\left(1+\operatorname{erf}\left[\left(E-E_{t h}\right) /\left(\sqrt{2} \sigma_{t h}\right)\right]\right.$ which gives an energy threshold of $E_{t h}=(19.7 \pm 0.1$ (stat. $\left.)\right) \mathrm{eV}$. This value corresponds to about $5.3 \sigma_{b}$ of the baseline noise. As expected, the width of the error function $\sigma_{t h}=(3.83 \pm 0.15) \mathrm{eV}$ is in agreement with the baseline noise $\sigma_{b}$ evaluated at $E=0$.

To fully exploit this improvement, a software-trigger algorithm for the continuously acquired data was developed. The trigger threshold was set accordingly to $19.7 \mathrm{eV}$. The entire data stream is processed with the optimum filter by an algorithm which avoids distortions in the finite Fourier transform (for details see [21]). An event is triggered whenever the filter output exceeds the chosen software threshold. Using the same filter for amplitude evaluation and triggering guarantees the consistency of the trigger definition and the energy calibration. The pulse data from hardware (standard DAQ) and software (continuous DAQ) trigger agree down to the hardware threshold of $\sim 40 \mathrm{eV}$. The pulse height evaluation of the optimum filter and the truncated standard event was studied on an event-by-event basis. In linear region of the pulse response, a maximum deviation of $2.8 \%$ is observed [14] which adds to the systematic error of the energy calibration. The final value of the threshold is $E_{t h}=(19.7 \pm 0.9) \mathrm{eV}$ including statistical and systematic errors. For the following DM analysis we use exclusively the data recorded with the continuous DAQ.

\subsection{Stability and data selection}

Since the data was not blinded for the calibration measurement [14], we choose the most conservative approach for this DM analysis: we apply no data quality cuts which might introduce an energy-dependent efficiency or might bias the result.

The only cut applied is the so-called stability cut which rejects periods where the detector is not in its correct operating point. The cut is based on the pulse height of control pulses (saturated pulses) which are continuously injected into the ohmic heater every $10 \mathrm{~s}$. This pulse height should remain constant if the operating point is stable. To be conservative all events within $\pm 5 \mathrm{~min}$ around every outlying control pulse are not used for the analysis. This removes $2.05 \mathrm{~h}$ from the total measuring time of $5.31 \mathrm{~h}$.

Figure 5 shows the stability of various detector parameters over the duration of the measurement. In the top frame the total event rate after the stability cut (grey crosses, left axis) 

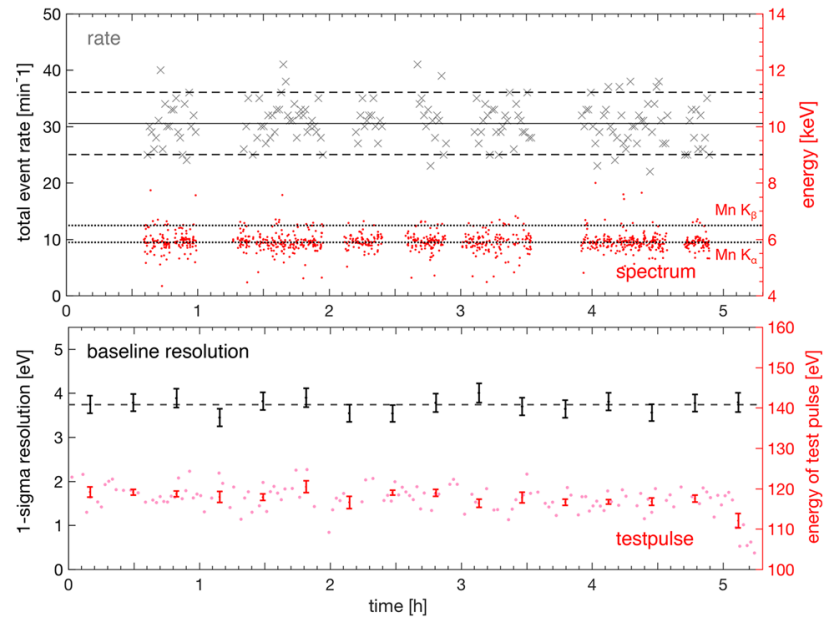

Fig. 5 Stability of the prototype detector over the measuring time of $5.31 \mathrm{~h}$ after the stability cut. Left y-axis: Total number of counts acquired per minute. Within statistics (dashed lines give Poissonian variance) the rate is stable over the entire run at a mean of $0.51 \mathrm{~Hz}$ (solid line). Right y-axis: Red dots show all events observed in the energy region of 4$8 \mathrm{keV}$. The positions of the $\mathrm{K}_{\alpha}$ and $\mathrm{K}_{\beta} \mathrm{X}$-ray energies (literature values) are indicated by dotted lines which are in agreement with the data. This demonstrates the stability of the detector response over time

and the energy of all events between 4 and $8 \mathrm{keV}$ (red dots, right axis) are depicted. The observed event rate is statistically consistent with a constant rate of $0.51 \mathrm{~Hz}$ (solid line). The dashed lines indicate the Poissonian variance $(1 \sigma)$. The dotted lines correspond to the literature values of the $\mathrm{K}_{\alpha}$ and $\mathrm{K}_{\beta}$ lines of ${ }^{55} \mathrm{Mn}$. Within statistics the positions of the X-ray lines are stable with time, demonstrating the stability of the energy calibration. The gaps in the data correspond to periods removed by the stability cut. The bottom frame shows the energy resolution as obtained from empty baseline samples (black error bars, left axis) and the energy of periodically injected test pulses corresponding to an energy of $\sim 120 \mathrm{eV}$ (red dots, right axis) together with their average ( $1 \sigma$ error bars). Over the entire measuring time the variance of the baseline is in agreement with $\sigma_{b}=(3.74 \pm 0.21) \mathrm{eV}$. The test pulse response also remains constant over time demonstrating again the stability of the detector response, except for the period at $5.0 \mathrm{~h}$ when the base temperature of cryostat has changed. This period is however removed by the stability cut (compare to upper frame of Fig. 5).

\section{Results and discussion}

We define the linear region of the pulse response which extends from the energy threshold of $19.7-600 \mathrm{eV}$ as our region-of-interest for DM search (ROI). The final spectrum is depicted in Fig. 2 (inset) with a binning of $5 \mathrm{eV}$. All 511 events in the ROI are considered conservatively as candidate events for DM particle-nucleus scattering, although it is assumed that these pulses originate from backgrounds. Since we do not apply data-quality cuts, ${ }^{2}$ the remaining events (higher energetic particle pulses, test pulses, control pulses and possible artifacts) are unspecified in the analysis. Again conservatively, we remove the sampling time $(614.28 \mathrm{~ms}$ per pulse) of the 5779 events which lie outside the ROI from the live-time. This results in a net live-time of $2.27 \mathrm{~h}$ which corresponds to a net exposure of $0.046 \mathrm{~g}$-days.

The constant background level of $\sim 1.2 \times 10^{5}$ counts $/(\mathrm{kg}$ $\mathrm{keV}$ day) is not unexpected due to lack of shielding against ambient radiation and due to the operation of the detector above ground. In addition, an X-ray calibration source was present during the entire measurement. The steep rise of the event rate towards threshold can be caused by source-related Auger electrons. Also, backgrounds such as beta/gamma or alpha decays on surfaces surrounding the detector could contribute to the exponentially increasing spectrum (see [14] for a more detailed discussion). The presence of exponentially rising, unknown backgrounds prohibits an unambiguous discovery of DM, however promising techniques are on their way to significantly reduce and understand remaining backgrounds [17].

Since the threshold of the prototype detector is lowered by more than one order of magnitude compared to previous macroscopic devices, the mass range of DM particles below $500 \mathrm{MeV} / \mathrm{c}^{2}$ can be probed for the first time despite a relatively high background level. An upper limit on the elastic spin-independent DM particle-nucleon cross section is derived. For the limit calculation we use the Yellin optimal interval method [22] which was developed to derive DM limits in the presence of backgrounds with unknown energy spectra. The sensitivity is based on a comparison of the observed spectrum [see Fig. 2 (inset)] and the expected recoil spectrum from DM particles of a certain mass $m_{\chi}$ in $\mathrm{Al}_{2} \mathrm{O}_{3}$.

For the calculation of the recoil spectrum we use the standard astrophysical parameters for the DM halo of the Milky Way: a Maxwellian velocity distribution, an asymptotic velocity of $220 \mathrm{~km} / \mathrm{s}$ and a galactic escape velocity of $544 \mathrm{~km} / \mathrm{s}$. The local DM density at Earth position is assumed to be $0.3 \mathrm{GeV} / \mathrm{cm}^{3}$. The Helm form factor [23] is used in the calculation of the scattering cross section. Both elements of $\mathrm{Al}_{2} \mathrm{O}_{3}$ are considered as targets. To take into account the finite energy resolution of the calorimeter the calculated spectrum is convolved with the Gaussian resolution $\left(\sigma_{b}=(3.74 \pm 0.21) \mathrm{eV}\right.$ at threshold).

The measurement presented here extends the reach of direct DM search experiments for $l \mathrm{DM}$ masses to below $500 \mathrm{MeV} / \mathrm{c}^{2}$. The achieved threshold of $E_{t h}=(19.7 \pm$ $0.9) \mathrm{eV}$ allows to probe elastic scattering of DM particles down to masses of $140 \mathrm{MeV} / \mathrm{c}^{2}$. Figure 6 shows the upper

\footnotetext{
${ }^{2}$ This implies that the cut efficiency is 1 all over the ROI.
} 


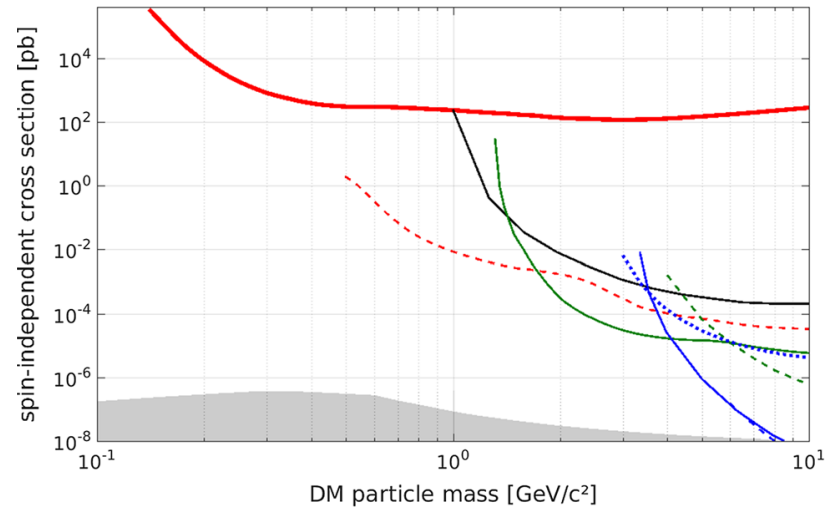

Fig. 6 Parameter space of DM mass vs. spin-independent DM particlenucleon cross-section. The result of this work (solid red) explores a new mass region between 140 and $500 \mathrm{MeV} / \mathrm{c}^{2}$ and extends the reach of DM direct searches. In comparison selected experimental results are shown: CRESST-II Phase 2 (dashed red) [16], CDMS-lite (full green) [24], EDELWEISS (dashed green) [25], XENON100 low mass analyis (dotted blue) [26], PandaX (full blue) [27], LUX (dashed blue) [28] and DAMIC (black) [29]. The shaded grey area indicates the neutrino-floor calculated for $\mathrm{Al}_{2} \mathrm{O}_{3}$

limit with $90 \%$ confidence level achieved in this work (red line). Below $500 \mathrm{MeV} / \mathrm{c}^{2}$ we explore a new region of parameter space via the direct detection of nuclear recoils and improve existing limits from the detection of bremstrahlung emission which accompanies nuclear recoils [12,13]. For masses below $\sim 1 \mathrm{GeV} / \mathrm{c}^{2}$ O-recoils dominate the recoil spectrum, while for higher masses Al-recoils provide the highest sensitivity. The transition is visible as a weak inflection in the exclusion curve.

In comparison, selected results of direct DM search experiments on the elastic spin-independent DM particle-nucleon cross-section are shown.

\section{Outlook}

Gram-scale cryogenic calorimeters have demonstrated their

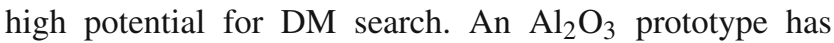
explored a new range of DM particles below $500 \mathrm{MeV} / \mathrm{c}^{2}$. This first result can be improved in two directions: (1) detector performance. A recently developed scaling law [14] predicts energy thresholds in the $1-10 \mathrm{eV}$ regime for cubic detectors of $\lesssim 1 \mathrm{~g}$. Modifications of the TES sensor are foreseen in order to reach this goal [14]. (2) Background level. The shielding of the setup used for the measurement can be improved significantly. We expect a reduction of the background rate by two orders of magnitude when installing a low-radioactivity $\mathrm{Pb}$ shielding around the experimental volume. A $4 \pi$ active cryogenic veto around the calorimeter would significantly reduce surface-related backgrounds (see
MC results in [17]) which are suspected to cause backgrounds at lowest energies.

The next generation of gram-scale calorimeters which will be developed for the $v$-cleus experiment [17] may extend the reach of direct search experiments to DM particle masses of $\mathcal{O}\left(10 \mathrm{MeV} / \mathrm{c}^{2}\right)$ and probe new models for DM. Due to hints that backgrounds at lowest energies are not dominated by cosmogenic radiation, large improvements in sensitivity may be achieved in above-ground or shallow laboratories.

Open Access This article is distributed under the terms of the Creative Commons Attribution 4.0 International License (http://creativecomm ons.org/licenses/by/4.0/), which permits unrestricted use, distribution, and reproduction in any medium, provided you give appropriate credit to the original author(s) and the source, provide a link to the Creative Commons license, and indicate if changes were made. Funded by $\mathrm{SCOAP}^{3}$.

\section{References}

1. G. Bertone, D. Hooper, J. Silk, Phys. Rep. 405(5-6), 279 (2005)

2. G. Jungman, M. Kamionkowski, K. Griest, Phys. Rep. 267(5-6), 195 (1996)

3. J. Ellis, T. Falk, K.A. Olive, M. Srednicki, Astropart. Phys. 13(2-3), $181(2000)$

4. B.W. Lee, S. Weinberg, Phys. Rev. Lett. 39, 165 (1977)

5. D.B. Kaplan, Phys. Rev. Lett. 68, 741 (1992)

6. K. Petraki, R.R. Volkas, Int. J. Mod. Phys. A 28(19), 1330028 (2013)

7. D.E. Kaplan, M.A. Luty, K.M. Zurek, Phys. Rev. D 79, 115016 (2009)

8. C. Bœhm, P. Fayet, Nucl. Phys. B 683(1-2), 219 (2004)

9. C. Boehm, T.A. Enßlin, J. Silk, J. Phys. G: Nucl. Part. Phys. 30(3), 279 (2004)

10. J.L. Feng, J. Kumar, Phys. Rev. Lett. 101, 231301 (2008)

11. R. Essig, A. Manalaysay, J. Mardon, P. Sorensen, T. Volansky, Phys. Rev. Lett. 109, 021301 (2012)

12. C. Kouvaris, J. Pradler, Phys. Rev. Lett. 118, 031803 (2017)

13. C. McCabe, Phys. Rev. D 96, 043010 (2017)

14. R. Strauss et al., Phys. Rev. D 96, 022009 (2017)

15. F. Pröbst et al., J. Low Temp. Phys. 100(1-2), 69 (1995)

16. G. Angloher et al., Eur. Phys. J. C 76(1), 25 (2016)

17. R. Strauss et al., Eur. Phys. J. C 77(8), 506 (2017)

18. G. Angloher et al., Astropart. Phys. 31, 270 (2009)

19. G. Angloher et al., Eur. Phys. J. C 74(12), 3184 (2014)

20. E. Gatti, P.F. Manfredi, Riv. Nuovo Cim. 9N1, 1 (1986)

21. G. Piperno, S. Pirro, M. Vignati, JINST 6, P10005 (2011)

22. S. Yellin, Phys. Rev. D 66, 032005 (2002)

23. J.D. Lewin, P.F. Smith, Astropart. Phys. 6, 87 (1996)

24. R. Agnese et al., Phys. Rev. Lett. 116(7), 071301 (2016)

25. L. Hehn et al., Eur. Phys. J. C 76(10), 548 (2016)

26. E. Aprile et al., Phys. Rev. D 94, 092001 (2016)

27. C. Fu et al., Phys. Rev. Lett. 118, 071301 (2017)

28. D.S. Akerib et al., Phys. Rev. Lett. 118, 021303 (2017)

29. A. Aguilar-Arevalo et al., Phys. Rev. D 94, 082006 (2016) 